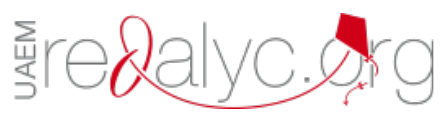

Centro Sur

ISSN: $2600-5743$

compasacademico@icloud.com

Grupo Compás

Ecuador

\title{
Diagnóstico de estrés laboral en los trabajadores de una empresa comercial
}

Álvarez Silva, Luis Antonio; Espinoza Samaniego, César Eduardo

Diagnóstico de estrés laboral en los trabajadores de una empresa comercial

Centro Sur, vol. 2, núm. 2, 2018

Grupo Compás, Ecuador

Disponible en: http://www.redalyc.org/articulo.oa?id=588861693005

Esta obra está bajo una Licencia Creative Commons Atribución-NoComercial-SinDerivar 4.0 Internacional. 


\section{Diagnóstico de estrés laboral en los trabajadores de una empresa comercial}

Diagnosis of work stress in the workers of a commercial company

Luis Antonio Álvarez Silva lalvarezsilva@uees.edu.ec Univesidad Especialidades Espíritu Santo, Ecuador

http://orcid.org/0000-0001-5835-714X

César Eduardo Espinoza Samaniego ceespinoza@uees.edu.ec Univesidad Especialidades Espíritu Santo, Ecuador

http://orcid.org/0000-0003-3748-0055

Centro Sur, vol. 2, núm. 2, 2018

Grupo Compás, Ecuador

Recepción: 19 Julio 2017 Aprobación: 14 Junio 2018

Redalyc: http://www.redalyc.org/ articulo.oa?id $=588861693005$

\section{BY-NC-ND}

Resumen: El presente estudio tiene como objetivo principal identificar el nivel de estrés laboral que tienen los trabajadores de una de las zonas mercantiles más antiguas y productivas de la ciudad de Guayaquil. La organización donde se realizó el estudio mantiene su anonimato, esta tiene como actividad económica la comercialización de indumentaria, la misma que posee alrededor de 10 locales de atención al público, la muestra fue de 243 trabajadores lo que representa un promedio del $95 \%$ de la organización. Se utilizó el Cuestionario de Estrés laboral de la OIT/OMS (Organización Internacional del Trabajo y la Organización Mundial de la Salud); en la aplicación del mismo se obtuvo un puntaje de 0.93 según la medida de consistencia interna alfa de Crombach. La conclusión del estudio señala que las personas que prestan sus servicios laborales a esta organización de la bahía de Guayaquil están expuestas a condiciones de trabajo recargadas tales como la alta cantidad de horas de trabajo, la escasez de días de descanso, el denso clima laboral, el liderazgo enfocado solamente en el resultado, por ende esta organización posee un alto nivel de estrés laboral; esto puede causar efectos negativamente potenciales en la salud de sus trabajadores.

Palabras clave: Estrés, Estrés Laboral, Organización, Trabajadores, diagnóstico.

Abstract: The main objective of this study is to identify the level of work stress experienced by workers in one of the oldest and most productive commercial areas of the city of Guayaquil. The organization where the study was carried out maintains its anonymity, this has as an economic activity the commercialization of clothing, the same one that has around 10 places of attention to the public, the sample was 243 workers, which represents an average of $95 \%$ of the organization. The ILO / WHO Work Stress Questionnaire (International Labor Organization and the World Health Organization) was used; in the application of the same, a score of 0.93 was obtained according to the Crombach alpha internal consistency measure. The conclusion of the study indicates that people who provide their labor services to this organization in the bay of Guayaquil are exposed to overcrowded working conditions such as the high number of working hours, the shortage of rest days, the dense work environment, the leadership focused only on the result, therefore this organization has a high level of work stress; This can have potentially negative effects on the health of your workers. In itself, these conditions can affect the achievement of the mission and the scope of the vision of the organization. Keywords: Stress, Work Stress, Organization, Workers, Working diagnosis.

\section{INTRODUCCIÓN}

A lo largo de la historia las actividades laborales del ser humano se han visto afectadas por múltiples características, estas acciones han 
desencadenado múltiples situaciones de bienestar y/o malestar en los individuos que la desempeñan (Carreño, Medina, Martínez, Juárez, y Vázquez, 2006). Además, estos factores han incitado a la aparición de fenómenos laborales, los cuales han tenido que ser estudiados por ser temas de interés científico y organizacional.

El trabajo demanda mucho tiempo de actividad en una persona, y es en este donde se originan múltiples sucesos en el individuo que lo realiza; estos terminan ocasionando desgaste en la salud, repercutiendo a una problemática que afecta significativamente a las personas en la actualidad (Gómez y Escobar, 2002). Por consiguiente, esta situación forma riesgos en las personas, unos de carácter físico y otros de carácter intangible; estos a su vez, tienen efectos negativos sobre el bienestar de los trabajadores; uno de esos desequilibrios es el estrés. Por tal razón, en la actualidad existen organizaciones que tienen identificados y relacionados los factores estresantes a la actividad laboral de los individuos (Sakuraya, y otros, 2017).

Siguiendo este orden de ideas, el estrés se presenta en todas las etapas de vida del ser humano, es decir, es una experiencia que los individuos distinguen a lo largo de su existencia, ya que los causantes del mismo pueden estar en cualquier escenario, sea este en el trabajo, la escuela o el hogar, ya que el estrés es un suceso potencial en el comportamiento de los individuos (Dich, Head, y Hulvej, 2016). El estrés ocasionado por el trabajo se denomina estrés laboral; este ha sido abordado desde un enfoque unidireccional, sin embargo esa característica de estudio lo estaría haciendo reduccionista, es por ello que el estudio del estrés laboral debe realizarse desde una perspectiva integral, en la que se engloben otros elementos, tal como la cultura organizacional (Ramos y Jordão, 2014).

Según la Organización Internacional del Trabajo (OIT), el estrés relacionado con el trabajo está determinado por la organización del trabajo, el diseño del trabajo, las relaciones laborales y tiene lugar cuando las exigencias del trabajo no corresponden o excedan a las capacidades, recursos o necesidades del trabajador; también se da, cuando el conocimiento y las habilidades de un trabajador no coinciden con las expectativas de la cultura organizativa de una empresa (OIT, 2016).

De esta forma, los sucesos de estrés laboral se volvieron un componente habitual y cotidiano en las personas que trabajan, en un mundo lleno de organizaciones hostiles enfocadas en la productividad a toda costa, en muchas ocasiones de forma injusta e inhumana, a tal punto que el estrés se convirtió en un fenómeno social, con implicaciones significativas que afectan el bienestar de los sujetos en las organizaciones (Amponsah y Annor, 2016).

Existen características que componen la organización del trabajo; estas son: la falta de un modelo de dirección con liderazgo, la sobrecarga de trabajo, la cultura empresarial cambiante, los problemas socioeconómicos, la intensidad y extensión de la jornada de trabajo, la necesidad de obtener mejores ingresos remunerativos, la interacción entre el individuo y su entorno; termina ocasionando lo que se denomina estrés laboral percibido (Avila, y otros, 2017). 
En este contexto, las organizaciones se vuelven escenarios sociales donde se presenta la interacción humana, la trasmisión de cultura, las condiciones de trabajo amenazantes, los bajos niveles de motivación, el desgaste de salud, los bajos niveles de rendimiento ( $\mathrm{Lu}$, y otros, 2017); siendo este escenario la condición de desarrollo del estrés laboral y es por ello la gran importancia de realizar diagnósticos en las organizaciones.

Por consiguiente, el presente estudio se realizará en una de las organizaciones más antiguas de la bahía de Guayaquil - Ecuador con más de 25 años de existencia; esta se encuentra ubicada en uno de los sectores de mayor incidencia mercantil. Esta fue la organización que proporcionó el acceso para la presente investigación, ya que la misma no se encuentra excluida de presenciar este tipo de fenómenos laborales. La razón social de esta empresa radica en la elaboración y comercialización de indumentaria y textiles en general, cuenta con talleres, oficinas administrativas, locales de venta directa y personal con varios años de trayectoria.

Por lo antes expuesto, es importante conocer el nivel de estrés laboral al que está expuesto el personal de esta empresa comercial de la bahía de Guayaquil, 2017. [1]

Desde el punto de vista de bienestar laboral, la Organización Internacional del Trabajo y la Organización Mundial de la Salud se han definido conocimientos que hacen énfasis a la terminología del estrés y los factores psicosociales, en la que se los presenta como aquellas interacciones que tiene: el trabajo, el ambiente laboral, los niveles de satisfacción, la organización como tal, las competencias del trabajador, la cultura organizacional; y como todos estos elementos pueden influir en la salud y en el rendimiento de determinado individuo que desempeña una actividad laboral (OIT, 1984).

Por esta razón, el estrés laboral se lo encasilla en la categoría de riesgo psicosocial, ya que concierne a las características de las condiciones de trabajo y de las organizaciones que pueden afectar la salud del individuo, ya sea en su parte psicológica o en su parte fisiológica. De tal manera, que ha sido un tema clave en las investigaciones, en la que se relacionan las características del trabajo y la salud de los individuos (Oshio, Inoue, y Tsutsumi, 2017).

Por tanto, este estudio presentará un acercamiento a la realidad de este fenómeno laboral en una organización, específicamente en el sector mercantil de la bahía de Guayaquil, la misma tendrá como objetivo principal diagnosticar el nivel de estrés laboral en sus trabajadores; para ello se analizarán los aspectos teóricos y culturales del estrés y el estrés laboral; además, se considerarán las características y actividades laborales principales de la compañía, por último, mediante levantamiento de información y método deductivo se realizará una síntesis con base en el resultado del nivel de estrés que posee la organización.

La palabra estrés es un derivado del latín Stringere que básicamente significa oprimir o apretar; por el siglo XVII esta palabra era utilizada para referir el sufrimiento o padecimiento que tenía una persona ante una determinada situación. Posteriormente en el siglo XVIII este término se 
lo relaciona con el vocablo inglés Strain cuyo significado alude a la tensión excesiva que comprime un material (Gómez y Escobar, 2002).

La definición de la palabra estrés tuvo varias opciones de abordaje, sin embargo a través de los siglos se pudo definir estrés como la fuerza o peso provocado por la presión (Thomae, 2002); un término muy relacionado con la Física. Por consiguiente, es importante aterrizar el término estrés a otros sectores de las ciencias, para poder comprender su forma teórica e integral.

Uno de los pioneros de la teoría del estrés fue el Doctor Hans Selye en 1926, quien reformuló desde los conocimientos de la Física un término de escala mundial y de impacto social, El término que utilizó fue "strain" que significa "tensión", el cual hace relación al desgaste y debilidad que sufren los materiales a través del tiempo, Selye relacionó que este proceso con características similares ocurren en los seres humanos, concibiendo la expresión stress que traducido al español es estrés; al cual lo definió como una respuesta biológica e inespecífica, del organismo ante cualquier demanda del medio exterior o un estado de tensión psicológica y fisiológica (Arias, 2012).

Desde un enfoque biológico y fisiológico, Selye indicaba que el estrés también se lo puede denominar Síndrome General de Adaptación, tema que hoy en día es multidimensionalmente aplicado a muchos factores de la sociedad, considerándose como un componente de la vida cotidiana (Cerezo, Hernández, Rodríguez, y Rivas, 2009).

Los estudios e investigaciones sobre el estrés siguieron extendiéndose. La amplitud de las publicaciones y algunos investigadores contemporáneos han seguido con los avances de la temática por medio de otros enfoques; por consiguiente sus aportes también han sido de gran incidencia en el campo de las ciencias médicas y ciencias sociales, dándose diferentes concepciones teóricas. Además, otro de los más destacados teóricos del estrés es Richard Lazarus, quien en 1999 presentó su teoría del estrés, las emociones y las valoraciones cognitivas desde un enfoque procesual o transaccional, contrastando con la teoría de Selye; bajo este enfoque se considera el aspecto del apoyo social como factor de protección ante el estrés (Shi, y otros, 2017).

Lazarus afirmaba que el estrés no es un resultado y que además un resultado psicológico o fisiológico no necesariamente es producto del estrés. En sus estudios, de modelo transaccional, se considera al estrés desde una perspectiva procesual (Ramos y Jordão, 2015), en este el estrés es un intervalo entre el individuo y la situación; además, en este modelo, no se considera al sujeto como un ente pasivo, más bien se reconoce que este es capaz de cognitivamente percibir la situación en función de su subjetividad. Del mismo modo, Lazarus afirmaba que el estrés no reside ni en la persona ni en el ambiente, sino en la interacción de ambos. (Ganster y Rosen, 2013)

Partiendo de este enfoque, para que exista estrés en un individuo es necesario que perciba de manera cognitiva un desequilibrio entre lo interno y lo externo, considerando su evaluación propia, el afrontamiento 
y que recursos se encuentran latentes para hacerle frente, por ello su nombre de modelo transaccional.

Definir la palabra Trabajo de forma universal y única sería muy complejo, ya que existen muchas razones que la intentan definir y estas son de diferentes enfoques. Por otra parte, el trabajo es parte de la vida de las personas y también de su calidad de vida (Torre y Rodríguez, 2013) y este se convierte en un constructo multidimensional que se basa en los valores significados y resultados que los individuos le dan a una actividad determinada.

El trabajo es una actividad común en el ser humano, a su vez es fuente de desarrollo personal, económico y social; en la cual el individuo no lo realiza de forma aislada sino en un medio social. Adicionalmente, la naturaleza del trabajo es vulnerable al cambio, más aún en los últimos años, es por ello que la satisfacción laboral y la tensión en el trabajo se han convertido parte de los medios laborales (Hoboubi, Choobineh, Kamari, Keshavarzi, y Akbar, 2016). De esta forma, el trabajo ha provocado sucesos considerados de gran incidencia en el bienestar y salud de los trabajadores, uno de estos fenómenos es el estrés laboral, ya que este se encuentra relacionado con el desarrollo del trabajo (Valadez, Bravo, y Vaquero, 2014).

Una de las incidencias y afectaciones más comunes que se presentan en los trabajadores son los problemas en las relaciones laborales, esta puede ser a causa de problemas sociales y de carácter turbulento (Orgambídez, Pérez, y Borrego, 2015). Por otra parte, la presencia de estrés laboral, puede generar desequilibrios en el organismo, desbalances bioquímicos, deterioro en el sistema corporal y uno de los de mayor impacto es el estrés en el trabajo por causa de las relaciones interpersonales, es decir por el fenómeno de subordinación y su dependencia, provocando desequilibrio en la armonía de la actividad laboral (Elfering, Gerhardt, Grebner, y Müller, 2016).

Las afectaciones por parte del estrés laboral, pueden ocasionar, problemas en las relaciones familiares, problemas de relaciones personales, problemas físicos, psicosomáticos, alteración en factores psicosociales tales como: la interacción en el trabajo, el medio ambiente, la satisfacción, la cultura, las necesidades, la situación fuera del trabajo, la percepción propia, todo esto en conjunto puede influir en la productividad y rendimiento de las personas. Por otro lado, el adecuado manejo de estas situaciones en el ámbito laboral proporciona un mayor control, mejor desempeño y permite que las personas sean menos propensas a experimentar prolongaciones de estrés laboral, agotamiento físico y mental, con lo que se podría tener un éxito laboral, siempre que este fenómeno se maneje de forma controlada (Shkoler y Tziner, 2017).

Dentro de las actividades laborales que el sujeto desempeña, existen situaciones permanentes en los cuales este debe interactuar con el medio desde una situación física y mental, a esta interacción se le denomina relación con los factores psicosociales, los cuales son de mucha relevancia a nivel de cultura organizacional, ya que contienen una temática de suma jerarquía como lo es el estrés laboral, el cual provoca grandes 
consecuencias a nivel individual y organizacional (Tziner, Rabenu, Radomski, y Belkin, 2015).

Desde un enfoque de la OMS; la salud y el estrés laboral se han venido definiendo por fenómenos organizacionales y sociales. Sin embargo, el estrés laboral es el resultado de la interacción entre los factores laborales, donde están inmersos el ambiente físico, las funciones biológicas, la cultura organizacional, los elementos psicosociales del trabajo y los que no son del trabajo, siendo el estrés laboral un efecto sobre el bienestar laboral, la productividad, el clima laboral, la seguridad y salud laboral (Krishnamurthy, y otros, 2016).

El estrés relacionado al trabajo o estrés laboral puede afectar el desempeño de los trabajadores provocando cambios en su comportamiento (Tziner y Sharoni, 2014). En la actualidad existen varias posturas críticas acerca del estrés laboral, desde el punto de vista humanista y desde el punto de vista organizacional, y es en estas dos categorías que se analiza el desgaste de la persona en el trabajo y también las bajas económicas que representa el estrés a nivel de empresas, debido a que el trabajo se ha convertido en la actividad transcendental de la existencia humana (Unda y otros, 2016).

El mayor de los organismos mundiales del trabajo, la Organización Internacional de Trabajo (OIT), proporciona un enunciado acerca de este fenómeno, señalando que el estrés laboral es la respuesta física y emocional a un daño provocado por la incompatibilidad entre las exigencias laborales y la capacidad del sujeto, además señala que el estrés relacionado con el trabajo está condicionado por la misma organización del trabajo, el esquema del trabajo, las relaciones laborales y se presenta cuando la demanda laboral excede la capacidad del individuo que la realiza o viceversa (OIT, 2016).

Son múltiples los artículos científicos sobre estrés laboral en documentos de alto nivel, ejemplo de ello es el caso de estudio de estrés laboral y personal en los recursos humanos de enfermería de una institución psiquiátrica de México, realizado por la Facultad de Medicina de la Universidad Autónoma de Querétaro, por medio de revisión documental y de literatura se llegó a la recomendación de conservar la salud mental mediante la práctica de mejores de estilos de vida (Basset, Estévez, Leal, Granados, y López, 2011). Por otra parte, tenemos el estudio de la reducción del estrés laboral en administrativos de la Universidad San Martín de Porres de Perú, en el cual la herramienta que se utilizó fue el cuestionario de la OIT/OMS para la detección de estrés laboral, en la misma se concluye que se debe realizar guías para la reducción del estrés laboral (Rivera, 2016).

En mención a la temática, también está el documento sobre el síndrome de estrés laboral crónico por el trabajo en los profesionales de la educación física brasileños del Centro de Investigación en Ciencias de la Salud por la Universidad Norte de Paraná, en el que se utilizó el inventario de Burnout Maslach, en esta se concluye que se debe intervenir e implementar cambios en los contextos del trabajo, destinados a mejorar la salud laboral y el bienestar (Dartagnan y Eron, 2016). 
Por tanto, a ritmo que la gestión empresarial avanza en un enfoque globalizador, las exigencias a los trabajadores y su fuerza laboral también aumentan, volviéndose objeto de estudio y escrutinio (Tziner y Sharoni, 2014). El estrés en el ámbito laboral es un fenómeno que puede desencadenarlo cualquier factor interno o externo del sujeto, además que es una temática de alta preocupación para los trabajadores por su gran complejidad (Li, y otros, 2014).

El estrés laboral según su intensidad y prolongación se puede convertir en destructivo y debilitante provocando daños en la salud de los individuos y de las organizaciones, ya que el bienestar en el trabajo está relacionada con el entorno laboral; además puede ser explicado como el punto de equilibrio entre el sujeto y el entorno, teniendo en consideración que el daño en el bienestar de los individuos por causa del estrés, afecta también a la organización, y en algunos de los casos el estrés puede llevar al suicidio por causa del trabajo, tal como detallan investigaciones realizadas en Japón (Honda, Date, Abe, Aoyagi y Honda, 2013).

En efecto, el estrés laboral presenta una problemática con repercusión negativa ante el individuo y las organizaciones, en lo cual está inmerso el estado de bienestar de la persona que realiza una actividad laboral, a tal punto que algunos países de Europa han tenido que detallar este fenómeno en sus legislaciones (Mucci, y otros, 2014); tal es su impacto, que la temática se multiplicó en diversos libros, revistas, artículos científicos, en tesis doctorales y de pregrado de Latinoamérica y el mundo, en ellos se aborda esta problemática como el origen de problemas organizacionales y de salud laboral.

El artículo 347 del código del trabajo de Ecuador, hace referencia a los riesgos ocasionados en el trabajo, a estos se los define como las eventualidades que ocasionan daños a un individuo mientras este realiza su actividad laboral. Por ello, es notorio que los daños no solo pueden ser físicos sino psicológicos, ya que la salud de un sujeto esta defina por tres ejes: lo físico, lo mental y lo social. (Ministerio de Trabajo, 2005).

En efecto, en el mes de junio del 2017, en nuestro país se proporcionó el acuerdo ministerial 20017-0082 en el respectivo registro oficial. En el artículo No. 9 de este acuerdo, proporcionado por el Ministerio de Trabajo, se manifiesta que toda organización pública y privada con más de 10 trabajadores debe implementar el programa de prevención de riesgos psicosociales. Este programa tiene como fin identificar, medir y controlar por medio de indicadores los factores que originan los riesgos psicosociales (Ministerio de Trabajo, 2017).

Tomando como referencia estos puntos, se considera que el estudio del fenómeno estrés laboral se encuentra encasillado en el estudio de riesgos psicosociales, por tal razón es de suma importancia el análisis de este fenómeno en la actualidad, sin embargo es notorio que este procedimiento de ley es contemporáneo en nuestro país.

Los modelos de medición, identificación y diagnostico obedecen a una base teórica ya validada a nivel internacional. Tal es el caso del modelo planteado por Robert Karasek quien planteo el enfoque demanda-control-apoyo, un modelo que recomienda un equilibrio entre 
las condiciones laborales y el individuo, en la que el control propio del sujeto es importante. Otro modelo fue el planteado por Siegrist, quien formuló el enfoque esfuerzo-recompensa, en este modelo se plantea que las recompensas económicas, la estima, la seguridad laboral proporcionan alternativas de solución ante el estrés laboral (Ceballos, Valenzuela, y Paravc, 2014).

Existen varios enfoques para el estudio de los fenómenos de estrés laboral, por consiguiente, también existen diferentes escalas de medición ante dicha temática. Uno de ellos es el Cuestionario de Maslach Burnout Inventory (MBI) (Maslach y Jackson, 1986), esta herramienta presenta altos niveles de confiabilidad con niveles oscilantes entre el 0.71 y 0.90 según la medida de consistencia interna Alfa de Crombach. Esta escala de fácil aplicabilidad cuenta con 22 ítems para ser contestados por las personas que realizan una respectiva actividad laboral.

La forma de contestación radica en una modalidad tipo Likert con 7 opciones de respuesta. Estas se distribuyen de la siguiente forma: 0 cuando la opción de respuesta es nunca, 1 cuando la opción de respuesta es pocas veces al año o menos; 2 una vez al mes o menos; 3 unas pocas veces al mes o menos; 4 una vez a la semana; 5 pocas veces a la semana; 6 todos los días. Tal como se detalla en la tabla No. 1 . Esta herramienta, realiza un estudio del estrés, cuando este se encuentra en un nivel alto, a lo que se denomina Burnout o síndrome del quemado, por tal razón hace énfasis en tres aspectos fundamentales que son: el agotamiento emocional, la despersonalización y la realización personal (Martínez, 2015).

Tabla 1.

Segmentos de Maslach Burnout Inventory (MBI)

\begin{tabular}{ll}
\hline Segmentos & No. Ítems \\
\hline $\begin{array}{l}\text { Cansancio } \\
\text { emocional }\end{array}$ & $1,2,3,6,8,13,14,16,20$ \\
\hline \begin{tabular}{l} 
Despersonalización \\
\hline $\begin{array}{l}\text { Realización } \\
\text { personal }\end{array}$
\end{tabular} & $4,10,11,15,22$ \\
\hline
\end{tabular}

Información sintetizada en el estudio. Elaboración: Autor.

Siguiendo este orden de ideas, los resultados en relación a razón de puntaje de medida de consistencia interna se detallan según los siguientes enunciados.

En España, 2013, un estudio de perfiles victimológicos en trabajadores acosados, tuvo una población de 2861 trabajadores españoles, para lo cual se utilizó el MBI para medir de forma exclusiva el estrés asistencial, para ello la medida de consistencia fue de 0.71 según alfa. Este estudio demostró que las víctimas de acoso laboral han presentado mayores situaciones clínicas y sintomatológicas (González-Trijueque y Graña, 2013).

En México, 2015, dos autores realizaron un estudio sobre estresores laborales y burnout en docentes de secundaria. La muestra fue de 28 docentes con una tipología de estudio de investigación-intervención. 
La medida de consistencia interna tuvo un promedio de 0.61 lo que la estimaría en aceptable, tomando de referencia que el análisis de confiabilidad por dimensión oscilo en $0.83,0.88$ y 0.12 . (Saltijeral y Ramos, 2015).

En España, 2015, un estudio de alto nivel estadístico que recababa la relación moderadora entre la autoeficacia, la autorregulación y el estrés laboral en una muestra de 106 profesores de primaria; se utilizaron varias herramientas de medición en la cual una de ellas fue el cuestionario breve de Burnout, que tiene los tres componentes de la herramienta del MBI; este puntuó un 0.74 de medida de consistencia interna según la medida de Alfa de Crombach (Tejedor y Lucas, 2016).

Tabla 2

Confiabilidad de la herramienta: Maslach Burnout Inventory (MBI), en diferentes estudios.

\begin{tabular}{lll}
\hline Autores & Año & $\begin{array}{l}\text { Alfa de } \\
\text { Crombach }\end{array}$ \\
\hline $\begin{array}{l}\text { González-Trijueque } \\
\text { y Graña }\end{array}$ & 2013 & 0,71 \\
\hline Saltijeral y Ramos & 2015 & 0,61 \\
\hline Tejedor y Lucas & 2015 & 0,74 \\
\hline
\end{tabular}

Información sintetizada en el estudio. Elaboración: Autor.

Por otra parte, se encuentra la Escala de estrés laboral de la OIT/ OMS la cual fue sustentada por Ivancevich y Matteson (Ivancevich y Matteson, 1989). Es un instrumento cuantitativo muy utilizado en países de Latinoamérica (Medina, Preciado, y Pando, 2007) para estudios de la misma particularidad, además por el respaldo que brinda la Organización Internacional del Trabajo y la Organización Mundial de la Salud para detectar estrés laboral (Suárez, 2013).

Esta escala cuenta con 25 ítems, que se encuentran relacionados a 7 segmentos de la actividad laboral, tal es el caso del clima organizacional, la estructura organizacional, la influencia del líder, la falta de cohesión, el territorio, la tecnología y el respaldo del grupo. La escala está diseñada según modelo Likert, en la cual existen siete (7) opciones de respuesta a cada ítem, las opciones oscilan en marcar con el número 7 si la respuesta es nunca, 6 si es en raras veces, 5 si es ocasionalmente, 4 si es en algunas veces, 3 si es frecuentemente, 2 si es generalmente y 1 si es siempre. Cada ítem analiza un segmento distinto, tal como se lo puede apreciar en la tabla 3. Además, esta escala cuenta con baremos que se aplican al resultado final, tal como se detalla en la tabla 4 . 
Tabla 3.

Dimensiones de la Escala de estrés laboral de la OIT/OMS

\begin{tabular}{cl}
\hline Segmentos & No. Ítems \\
\hline Clima & $1,10,11$, \\
organizacional & 20 \\
\hline Estructura & $2,12,16$, \\
organizacional & 24 \\
\hline Territorio & $3,15,22$ \\
organizacional & \\
\hline Tecnología & $4,14,25$ \\
\hline Influencia del & $5,6,13$, \\
líder & 17 \\
\hline Falta de & $7,9,18$, \\
cohesión & 21 \\
\hline Respaldo del & $8,19,23$ \\
grupo & \\
\end{tabular}

Información sintetizada en el estudio. Elaboración: Autor.

Tabla 4

Baremo - Rango de niveles de estrés laboral de la escala

\begin{tabular}{ll}
\hline $\begin{array}{l}\text { Alto nivel de } \\
\text { estrés }\end{array}$ & $<90,2$ \\
\hline Estrés & $90,3-117,2$ \\
\hline $\begin{array}{l}\text { Nivel } \\
\text { intermedio }\end{array}$ & $117,3-153,2$ \\
\hline $\begin{array}{l}\text { Bajo nivel de } \\
\text { estrés }\end{array}$ & $>153,3$ \\
\hline
\end{tabular}

Información sintetizada en el estudio. Elaboración: Autor

El cuestionario en mención, ha sido utilizado en varias ocasiones y en diferentes ámbitos laborales, tal es el caso del estudio de adaptación de la escala de estrés laboral para trabajadores mexicanos en el 2007, esta utilizó una muestra de 38072 trabajadores la cual tuvo un nivel de confiabilidad según alfa de 0.92 (Medina, Preciado, y Pando, 2007). En el 2009, un estudio realizado en Venezuela, se enfocó en el análisis de las características del estrés en trabajadores de un hospital, en el cual la muestra fue de 67 trabajadores y cuyo nivel de confiabilidad fue de 0.91 según alfa (Sanabria y Rojas, 2009).

En el 2013, se realizó la aplicación de la escala de estrés laboral, a trabajadores de una central telefónica de Perú, el mismo que utilizó una muestra de 203 trabajadores en el cual el nivel de confiabilidad obtuvo un puntaje de 0.97 (Suárez, 2013). En 2015, se realizó un estudio de estrés laboral usando como herramienta la escala de la OIT-OMS, la misma que fue proporcionada a una muestra de 115 profesores de una secundaria pública, este brindo un coeficiente de consistencia interna de 0.94 según alfa (Torres, 2015).

Por otra parte, en 2016, se realizó una investigación tipo experimental en el cual se analizó la efectividad del mate de coca en la reducción del estrés laboral, para lo cual se utilizó la escala de la OIT/OMS en una muestra experimental de 10 personas y una muestra de control de 10 
personas, este estudio presento un nivel de confiabilidad de 0.96 según alfa (Rivera, 2016). En la siguiente tabla explicativa se muestra una síntesis de los niveles de confiabilidad que se han obtenido por la aplicación de la escala de estrés laboral de la OIT/OMS en diferentes estudios.

Tabla 5.

Confiabilidad de la herramienta: Cuestionario de Estrés Laboral de la OIT/OMS, en diferentes estudios.

\begin{tabular}{lll}
\hline Autores & Año & $\begin{array}{l}\text { Alfa de } \\
\text { Crombach }\end{array}$ \\
$\begin{array}{l}\text { Medina, } \\
\begin{array}{l}\text { Preciado, } \\
\text { y Pando }\end{array}\end{array}$ & 2007 & 0,92 \\
$\begin{array}{l}\text { Sanabria, } \\
\text { C.,y }\end{array}$ & 2009 & 0,91 \\
$\begin{array}{l}\text { Rojas, L. } \\
\text { Suárez, }\end{array}$ & 2013 & 0,92 \\
$\begin{array}{l}\text { A. } \\
\text { Torres, } \\
\text { M. }\end{array}$ & 2015 & 0,94 \\
$\begin{array}{l}\text { Rivera, } \\
\text { M. }\end{array}$ & 2016 & 0,96 \\
\end{tabular}

Información sintetizada en el estudio. Elaboración: Autor.

Por otra parte, con respecto al tema de validez del cuestionario de estrés laboral de la OIT/OMS, se demostraron altos niveles. En el estudio del 2007, de adaptación de la escala a un grupo de trabajadores mexicanos se obtuvo una validez de nivel adecuado, esto se realizó por medio de análisis factorial confirmatorio en el cual la prueba KMO fue de 0.91, la de esfericidad fue de 2583.93 y 0.000 de significancia (Medina, Preciado, y Pando, 2007).

En el estudio de estrés laboral, del 2009, realizado a trabajadores de un hospital de Venezuela, se obtuvo la validez del cuestionario por medio de la validez formal de contenido (Sanabria y Rojas, 2009). Por otra parte, en 2013, el estudio de adaptación de la escala a trabajadores peruanos de una central de llamadas obtuvo una validez de contenido a través de criterio de jueces utilizando el análisis binomial en la que los resultados fueron menores a 0.05 , mostrando el acuerdo y concordancia de los expertos, además se realizó una validez de constructo por medio de análisis factorial en la que se obtuvo que la prueba $\mathrm{KMO}$ es de 0.953 y la prueba de esfericidad es de 4752.595 (Suárez, 2013).

Siguiendo este orden de ideas, en el estudio de estrés laboral realizado a profesores mexicanos en 2015, se determinó la validez de contenido del instrumento por medio de prueba piloto a profesores que no formaron parte de la muestra (Torres, 2015). Por último, en el estudio experimental de estrés laboral realizado a trabajadores peruanos en el 2016, evidencío que el cuestionarlo de estrés laboral de la OIT/OMS presenta una validez de contenido, basándose en los estudios realizados en demás partes de Perú (Rivera, 2016). En la siguiente tabla se presenta una síntesis de la validez de la herramienta por diferentes autores. 
Tabla No. 6

Validez de la herramienta por diferentes estudios.

\begin{tabular}{lcl}
\hline Autores & Año & $\begin{array}{l}\text { Tipo de } \\
\text { validez }\end{array}$ \\
\hline $\begin{array}{l}\text { Medina, } \\
\text { Preciado, y } \\
\text { Pando. }\end{array}$ & 2007 & $\begin{array}{l}\text { Validez de } \\
\text { constructo. }\end{array}$ \\
\hline $\begin{array}{l}\text { Sanabria, } \\
\text { C., y Rojas, } \\
\text { L. }\end{array}$ & & $\begin{array}{l}\text { Validez de } \\
\text { contenido. }\end{array}$ \\
\hline Suárez, A. & 2013 & $\begin{array}{l}\text { Validez de } \\
\text { contenido } \\
\text { y de } \\
\text { Constructo. }\end{array}$ \\
\hline Torres, M. & 2015 & $\begin{array}{l}\text { Validez de } \\
\text { Contenido. }\end{array}$ \\
\hline Rivera, M. & 2016 & $\begin{array}{l}\text { Validez de } \\
\text { contenido. }\end{array}$ \\
\hline
\end{tabular}

Información sintetizada en el estudio. Elaboración: Autor.

Siguiendo este orden de ideas, el presente estudio tomará como referencia la utilización de la herramienta de estrés laboral de la OIT/ OMS, por su gran impacto en el medio latino y por sus altos puntajes de validez y confiabilidad.

La organización

La organización objeto de estudio presenta 25 años de existencia en el mercado ecuatoriano, su misión radica en la fabricación y comercialización de vestimenta para las personas, en la actualidad está presente en una de las zonas más comerciales y populares de la ciudad de Guayaquil, esta es la bahía. En mencionada zona, presenta 10 locales de atención al cliente y venta directa, bodegas internas y externas, talleres y una oficina administrativa. La distribución aproximada es de 20 trabajadores por cada local de atención al cliente.

El logro de la misión de la organización obedece a una serie de planificaciones internas realizadas, a través de los años, sin embargo las modalidades de trabajo, los horarios de labores, la carga, la centralización de actividades, la división del trabajo y la toma de decisiones, ha influenciado en la compañía según los análisis de las consultorías realizadas; de tal manera que las características organizacionales de la compañía obedecen a un modelo mecanicista (Amaru, 2009).

\section{MATERIALES Y MÉTODOS}

En este estudio se analizaron los componentes que se anexan al desarrollo del estrés laboral, es decir que el abordaje del estrés laboral es desde una perspectiva resultadista en el que intervienen varios factores. Por otra parte, el diseño metodológico se compone de tres partes que son: el enfoque, el proceso y el método. Se parte de la representación que el enfoque es el modelo de pensamiento que se sigue en el estudio, por ello 
el enfoque de este estudio es empírico analítico ya que permite describir la realidad (Duque, 2014).

La presente investigación es de carácter No experimental tipo transversal, además presenta un alcance descriptivo, las características del proceso son cuantitativas tal como señala el modelo de la metodología de Roberto Hernández Sampieri (Hernández, Fernández, y Baptista, 2010). El método o tipo de razonamiento a utilizar es de carácter deductivo debido a que se abordará el estudio del fenómeno estrés laboral desde lo general a lo particular, es decir de las afectaciones del fenómeno a nivel organizacional direccionado a lo individual.

La muestra de esta investigación obedece a las características del muestreo no probabilístico tipo intencional, el cual se ajusta al criterio del investigador (Hernández, Fernández, y Baptista, 2010), por ende, se consideró el personal de la organización que tiene un tiempo determinado de labores ininterrumpidas; es decir, el personal que tiene laborando mayor-igual a 1 año de actividades; esto representa el 95\% del personal de la organización, que según la nómina del área de recursos humanos de la compañía, son 243 trabajadores.

Las características de la muestra son las siguientes: están presentes en tres de las áreas de la organización, estas son: comercial el 78\%, operaciones el $18 \%$ y administrativo el $4 \%$. La cantidad de hombres es del $16 \%$ y de las mujeres de $85 \%$. El nivel de instrucción es: básica el $37 \%$, bachillerato el $60 \%$, superior en curso el $2 \%$ y con estudios superiores terminados el $1 \%$. Adicional a la problemática, el personal en mención no ha recibido capacitación en los últimos 2 años, por temas presupuestarios, por tal razón se confirmó que no conocen normativas de procesos, objetivos organizacionales, de seguridad y salud ocupacional. 
Tabla No. 7.

Características de la muestra.

\begin{tabular}{|c|c|c|}
\hline \multicolumn{3}{|c|}{$\begin{array}{l}\text { Cantidad de Personal de la } \\
\text { organización }\end{array}$} \\
\hline 255 & & $100 \%$ \\
\hline \multicolumn{3}{|c|}{$\begin{array}{l}\text { Cantidad de personal de la } \\
\text { organización }>=\text { a } 1 \text { año de } \\
\text { labores }\end{array}$} \\
\hline$n=243$ & & $95 \%$ \\
\hline \multicolumn{3}{|c|}{ Distribución por género } \\
\hline & $\begin{array}{l}\text { \# de } \\
\text { personal }\end{array}$ & $\begin{array}{l}\text { \% de } \\
\text { personal }\end{array}$ \\
\hline Masculino & 38 & $15 \%$ \\
\hline Femenino & 205 & $85 \%$ \\
\hline \multicolumn{3}{|c|}{ Áreas de la Organización } \\
\hline & $\begin{array}{l}\text { \# de } \\
\text { personal }\end{array}$ & $\begin{array}{l}\text { \% de } \\
\text { personal }\end{array}$ \\
\hline Comercial & 189 & $78 \%$ \\
\hline Operaciones & 44 & $18 \%$ \\
\hline Administrativo & 10 & $4 \%$ \\
\hline \multicolumn{3}{|c|}{ Nivel de instrucción } \\
\hline Básica & 90 & $37 \%$ \\
\hline Secundaria & 146 & $60 \%$ \\
\hline $\begin{array}{l}\text { Superior en } \\
\text { curso }\end{array}$ & 5 & $2 \%$ \\
\hline $\begin{array}{l}\text { Superior } \\
\text { terminado }\end{array}$ & 2 & $1 \%$ \\
\hline \multicolumn{3}{|c|}{ Rango de edad } \\
\hline 18-30 años & 150 & $62 \%$ \\
\hline 31-40 años & 61 & $25 \%$ \\
\hline 41-50 años & 17 & $7 \%$ \\
\hline $51->$ años & 15 & $6 \%$ \\
\hline \multicolumn{3}{|l|}{ Estado civil } \\
\hline Soltero/a & 92 & $38 \%$ \\
\hline Unión libre & 87 & $36 \%$ \\
\hline Casadola & 55 & $22 \%$ \\
\hline Divorciado/a & 7 & $3 \%$ \\
\hline Viudo/a & 2 & $1 \%$ \\
\hline
\end{tabular}

Información sintetizada en el estudio. Elaboración: Autor.

La edad de los trabajadores oscila entre 18 a 30 años el 62\%, de 31 a 40 años el 25\%, de 41 a 50 años el $7 \%$ y de 51 años en adelante el 6\%. Los estados civiles de los involucrados son: solteros el $38 \%$, en unión libre el $36 \%$, casados el $22 \%$, divorciados el $3 \%$ y en viudez el $1 \%$.

Para efectuar el procedimiento de levantamiento de información, se solicitó los permisos correspondientes a la gerencia y los empleados de la organización, quienes proporcionaron la aceptación del estudio, por ende se realizó un proceso de comunicación organizacional sobre la fecha de la aplicación y además se brindó los beneficios legales y sociales de la implementación de la metodología. Por consiguiente, se procedió a efectuar el levantamiento de información en el mes de febrero del presente año; esto se dio por cada sección comercial, operativa y administrativa en forma segmentada, reunida y por medio de la herramienta en físico; todo esto para no influir en su jornada de tiempo de labores y en consideración que la gran mayoría de los colaboradores no tiene acceso a un ordenador debido a las características del trabajo. Una vez obtenida la información 
de la muestra se procedió a ingresar los datos en el programa Microsoft Excel y SPSS versión 22.

\section{RESULTADOS}

La validación metodológica de los resultados, proporcionados por los datos de recopilación por medio de la herramienta, se realizó mediante análisis de confiabilidad y validez estadística.

El análisis de confiabilidad de los resultados se lo realizó por medio de la medida de consistencia interna Alfa de Crombach, con el que se obtuvo una medida de 0.93, que según Metodología de la investigación de Hernández Sampieri es un puntaje alto (Hernández, Fernández, y Baptista, 2010). La validez del instrumento se lo obtuvo por medio de la medida Kaiser-Meyer-Olkin (KMO) con lo que se obtuvo un puntaje de 0.92, una puntuación alta. La prueba de esfericidad de Bartlett es de 0.00; las comunalidades tienen un promedio de 0.597.

Con lo antes indicado, se puede señalar que la herramienta tiene altos niveles de confiabilidad y validez de aplicación en nuestro medio. Los resultados obtenidos oscilan en relación a las 7 dimensiones de estudio del cuestionario.

En la Dimensión Clima Organizacional (CLIORG) que comprende los ítems 1,10, 11 y 20; se obtuvo una medida de confiabilidad superior a 0.68. La interpretación de los resultados radica en que el $62 \%$ de los encuestados señalaron que el ambiente general de trabajo es motivo de estrés laboral. En la dimensión Estructura Organizacional (ESTORG) que comprende los ítems 2, 12, 16 y 24; se obtuvo una medida de confiabilidad superior a 0.55 . Por parte de los encuestados el $61 \%$ considera que la estructura organizacional en la que incluye su organigrama y línea de mando es causante de estrés laboral.

La dimensión de Territorio Organizacional (TERORG) obtuvo una medida de 0.52 de consistencia interna considerándose nivel medio en este estudio, adicionalmente el $70 \%$ de los encuestados afirma que la ubicuidad de la organización no es fuente de estrés, colocándolo como una característica no influyente en el presente estudio.

La dimensión de Tecnología (TECNO) que hace referencia a los ítems 4, 14 y 25 tiene una medida de consistencia interna de 0.55 . Por consiguiente dentro de la interpretación de los resultados se obtiene que el $60 \%$ de los encuestados considera que la poca utilización de la tecnología no permite avanzar a una competitividad comercial de alto nivel, ocasionando esto malestar y desgaste en el personal.

La dimensión Líder (LIDER) que hace referencia a los ítems 5, 6, 13 y 17 obtuvo una medida de consistencia interna de 0.76 . Adicionalmente el porcentaje del personal que considera que la influencia del líder, en este caso el supervisor o jefe inmediato, interviene en su estado de estrés laboral es del 69\%. La Dimensión Cohesión (FCOHE) que hace referencia los ítems 7, 9, 18 y 21 obtuvo una medida de consistencia interna de 0.77 . Adicionalmente se obtuvo que el $67 \%$ del personal encuestado confirma 
que la relación entre pares, la falta de organización grupal es fuente de estrés laboral.

La Dimensión Grupo (RESGRUP) que hace referencia a los ítems 8, 19 y 23 presento un nivel de consistencia interna de 0.74 . Por consiguiente, el 69\% del personal encuestado afirma, al igual que la dimensión anterior, que el conocimiento técnico del grupo y la ayuda que este no proporcione es fuente de estrés laboral.

En fin, la Organización Mundial de la Salud y la Organización Internacional del Trabajo al presentar su baremo de puntajes, señalan una ponderación sobre los indicadores de nivel de estrés, tal como se señala en la tabla 4 del presente estudio, por tal razón el nivel de estrés laboral de la organización es de: 78 (presenta un alto nivel de estrés laboral)

Tabla 8

Índice de estrés laboral de la organización

\begin{tabular}{ll}
\hline Muestra & $\begin{array}{l}\text { Índice de } \\
\text { estrés laboral }\end{array}$ \\
\hline $\mathrm{n}=243$ & $\begin{array}{l}78 \text { (alto nivel de } \\
\text { estrés) }\end{array}$ \\
\hline
\end{tabular}

Información sintetizada en el estudio. Elaboración: Autor.

\section{DISCUSIÓN}

La herramienta como tal es patrocinada por dos de los entes más incidentes del mundo a nivel de Salud y condición Laboral, sin embargo esta no es muy considerada a nivel mundial, pero en América latina tiene un mayor grado de acogida. Sin embargo, también es importante señalar que otra gran cantidad de estudios sobre el fenómeno de estrés laboral se los estudia como una parte de los riesgos psicosociales, es por ello que en la gran mayoría de estudios de esta temática, se utiliza la metodología de la herramienta española FPSICO (Ferrer, Guilera, y Peró, 2011).

Con respecto al proceso de confiabilidad y validez de la herramienta se encuentra determinada cantidad de información de la misma en las revistas de investigación y divulgación científica. Sin embargo, es notorio que dentro del análisis que se tiene de los diferentes autores, que han utilizado la herramienta, esta ha puntuado un alto nivel según la medida de consistencia interna; ubicándose en un promedio de 0.93 según alfa de Crombach y un nivel de validez adecuado según los análisis factoriales respectivas.

En el análisis de confiablidad de las dimensiones, los niveles tienden a disminuir, tal como se pudo identificar en la parte de análisis de los resultados, sin embargo no se rechazan ya que según la teoría, lo niveles superiores a 0.5 son aceptables (Hernández, Fernández, y Baptista, 2014). La presentación original de la herramienta, con su traducción del inglés no presenta un correcto entendimiento con base al léxico latino, por ello es necesario adaptar ciertos palabras para su mejor entendimiento, tal es el caso de los ítems 1,6 y 12. 
Además, existe bibliografía que hace mención a la utilización de la herramienta, pero originalmente de países como Perú, Venezuela y México. Sin embargo, existe otra gran cantidad de información que se tiene de los estudios por medio de la herramienta, pero esta reposa en investigaciones de biblioteca que son utilizadas para los procesos de titulación de estudiantes de pregrado académico.

Por último, existen puntajes en la etapa de validez de la herramienta en que el índice de comunalidades de los ítems 2,3 y 8 proporcionan un puntaje promedio de 5.05; esto se acepta según la teoría, sin embargo esta en un límite de no cumplir con este requisito.

\section{Referencias}

Amaru, A. (2009). Fundamentos de Administración, teoría general y proceso administrativo. México: Pearson educación.

Amponsah, K., y Annor, F. (2016). Do Personality and Organizational Politics Predict Workplace Victimization? A Study among Ghanaian Employees. Safety and Health at Work, 72-76.

Arias, W. (2012). Estrés laboral en trabajadores desde el enfoque. Revista Cubana de Salud Pública, 525-535.

Avila, I., De Nazelle, A., Cole, T., Donaire, D., Jerrett, M., Rodriguez, D., y Nieuwenhuijsen, M. (2017). The relationship between bicycle commuting and perceived stress: a crosssectional study. BMJ Open, 1-11.

Basset, I. I., Estévez, R., Leal, M., Granados, G., y López, J. (2011). Estrés laboral y personal en los recursos humanos de Enfermería de una Unidad de Psiquiatría en México, D.F. Enfermería Neurológica, 27-31.

Carreño, S., Medina, M., Martínez, N., Juárez, F., y Vázquez, L. (2006). Características organizacionales, estrés y consumo de alcohol en trabajadores de una empresa textil mexicana. Salud mental, 63-70.

Ceballos, P., Valenzuela, S., y Paravc, T. (2014). Factores de riesgos psicosociales en el trabajo: género y enfermería. Avances en enfermería, 271-279.

Cerezo, S., Hernández, M., Rodríguez, G., y Rivas, R. (2009). Efectos de una intervención de manejo de estrés en mujeres con hipertensión arterial sistémica. Acta Colombiana de Psicologia, 85-93.

Dartagnan, P., y Eron, J. (2016). Síndrome de estrés laboral crónico por el trabajo (burnout) en los profesionales de la educación física brasileños. Revista de Psicologia del Deporte, 253-260.

Dich, N., Head, J., y Hulvej, N. (2016). Role of psychosocial work factors in the relation between becoming a caregiver and changes in health behaviour: results from the Whitehall II cohort study. Epidemiol Community Health, 1200-1206.

Duque, J. (2014). Planteamiento, desarrollo y divulgación de resultados en proyectos de investigación: Un enfoque aplicado a las ciencias de gestión: Diseño metodológico general. En E. J. Oliva, Investigación en Ciencias de Gestión (pág. 55). Bogotá: Universidad Autónoma de Colombia.

Elfering, A., Gerhardt, C., Grebner, S., y Müller, U. (2016). Exploring Supervisor-Related Job Resources as Mediators between Supervisor Conflict and Job Attitudes in Hospital Employees. Safety and Health at Work, 19-28. 
Ferrer, R., Guilera, G., y Peró, M. (2011). Propiedades Psicométricas del Instrumento de Valoración de Riesgos Psicosociales del INstituto Nacional de Seguridad e Higuiene en el trabajo (FPSICO\}. Barcelona: Universitat de Barcelona.

Ganster, D. C., y Rosen, C. C. (2013). Work Stress and Employee Health: A Multidisciplinary Review. Journal of Management, 1085-1122.

Gómez, B., y Escobar, A. (2002). Neuroanatomía del estrés. Revista Mexicana de Neurociencia, 273-282.

Gómez, V. (2005). Richard Stanley Lazarus. Revista Latinoamericana de Psicología, 207-209.

González-Trijueque, D., y Graña, J. (2013). Perfiles victimológicos en trabajadores acosados en su lugar de trabajo: Niveles de psicopatología y burnout. Revista Argentina de Psicología Clínica, 121-130.

Hernández, R., Fernández, C., y Baptista, M. (2014). Metodología de la Investigación Cientifica 5ta Edición. México: McGraw-Hill.

Hoboubi, N., Choobineh, A., Kamari, F., Keshavarzi, S., y Akbar, A. (2016). The Impact of Job Stress and Job Satisfaction on Workforce Productivity in an Iranian Petrochemical Industry. Safety and Health at Work, 67-71.

Honda, A., Date, Y., Abe, Y., Aoyagi, K., y Honda, S. (2013). Workrelated Stress, Caregiver Role, and Depressive Symptoms among Japanese Workers. Safety and Health at Work, 7-12.

Ivancevich, J., y Matteson, M. (1989). Estrés y trabajo: una perspectiva gerencial . México: Trillas.

Krishnamurthy, M., Ramalingam, P., Perumal, K., Perumal, L., Chinnadurai, J., Shanmugam, R., ... Venugopal, V. (2016). Occupational Heat Stress Impacts on Health and Productivity in a Steel Industry in Southern India. Safety and Health at Work, 99-104.

Li, L., Hu, H., Zhou, H., He, C., Fan, L., Liu, X., .. . Sun, T. (2014). Work stress, work motivation and their effects on job satisfaction in community health workers: a cross-sectional survey in China. BMJ Open, 1-9.

Lu, Y., Hu, X.-M., Huang, X.-L., Zhuang, X.-D., Guo, P., Feng, L.-F., . . Tao Hao, Y. (2017). The relationship between job satisfaction, work stress, work-family conflict, and turnover intention among physicians in Guangdong, China: a cross-sectional study. BMJ Open, 1-12.

Martínez, P. (2015). Cómo se defiende el profesorado de secundaria del estrés: burnout y estrategias de afrontamiento. Journal of Work and Organizational Psychology, 1-9.

Maslach, C., y Jackson, S. (1986). Maslach Burnout Inventory (2nd Ed). Palo Alto, CA: Consulting Psychologists Press.

Medina, S., Preciado, M., y Pando, M. (2007). Adaptación de la escala de estrés laboral organizacional para trabajadores mexicanos. Respyn. Obtenido de http://www.respyn.uanl.mx/viii/4/articulos/escala_estres.htm

Ministerio de Trabajo. (2005). Código de Trabajo. Quito: Lexis.

Ministerio de Trabajo. (2017). Acuerdo Ministerial No. Mdt-2017-0082, Expedir la normativa para la erradicación de la discriminación en el ámbito laboral. Quito: Lexis.

Mucci, N., Giorgi, G., Cupelli, V., Gioffrè, P., Rosati, M., Tomei, F., . . . Arcangeli, G. (2014). Work-related stress assessment in a population 
of Italian workers. The Stress Questionnaire. Science of the Total Environment, 673-679.

OIT. (1984). Informe del Comité Mixto OIT/OMS de Medicina del Trabajo. Ginebra: OIT.

OIT. (2016). Estrés en el trabajo: un reto colectivo. Ginebra: Publicaciones de la OIT .

Orgambídez, A., Pérez, P., y Borrego, Y. (2015). Estrés de rol y satisfacción laboral: examinando el papel mediador del engagement en el trabajo. Journal of Work and Organizational Psychology, 69-77.

Oshio, T., Inoue, A., y Tsutsumi, A. (2017). Examining the mediating effect of workto-family conflict on the associations between job stressors and employee psychological distress: a prospective cohort study. BMJ Open, $1-10$.

Ramos, V., y Jordão, F. (2014). La relación entre el estrés laboral, las fuentes que le dan origen y las estrategias de coping en el sector público y el privado. Journal of Work and Organizational Psychology, 11-20.

Ramos, V., y Jordão, F. (2015). Relación entre el conflicto de valores, el sector y el estrés laboral: un modelo descriptivo. Psicología Iberoamericana, 16-27.

Rivera, M. (2016). Efectividad del uso del mate de coca en la reducción del estrés laboral en administrativos de una universidad. Horizonte Médico, 20-28.

Sakuraya, A., Watanabe, K., Kawakami, N., Imamura, K., Ando, E., Asai, Y., ... Tsutsumi, A. (2017). Work-related psychosocial factors and onset of metabolic syndrome among workers: a systematic review and meta analysis protocol. BMJ Open, 1-5.

Saltijeral, M., y Ramos, L. (2015). Identificación de estresores laborales y burnout en docentes de una secundaria para trabajadores del Distrito Federal. Salud Mental, 361-369.

Sanabria, C., y Rojas, L. (2009). Caracterización del estrés en trabajadores de un hospital Infantil universitario, como estrategia para evaluar la Competitividad organizacional. Revista del Centro de Investigación de Ciencias Administrativas y Gerenciales, 1-14.

Shi, L., Wang, L., Jia, X., Li, Z., Mu, H., Liu, X., ... Fan, L. (2017). Prevalence and correlates of symptoms of post-traumatic stress disorder among Chinese healthcare workers exposed to physical violence: a cross crosssectional. BMJ Open, 7(7), 1-11.

Shkoler, O., y Tziner, A. (2017). The mediating and moderating role of burnout and emotional. Journal of Work and Organizational Psychology, 33(2), 157-164.

Suárez, Á. (2013). Adaptación de la Escala de estrés Laboral de la OIT-OMS. Revista PsiqueMag,2(1), 33-50.

Tejedor, E., y Lucas, S. (2016). La autoeficacia y la autorregulación como variables moderadoras del estrés laboral en docentes de educación primaria. Universitas Psychologica, 15(1),205-218.

Thomae, H. (2002). Haciéndole frente al estrés en la vejez. Revista Latinoamericana de Psicología, 34(1-2),42-54.

Torre, M. D., y Rodríguez, R. (2013). Psicoterapia del acoso psicológico en el trabajo. Revista Argentina de Clínica Psicológica, 22(2),131-138.

Torres, M. (2015). Estrés y condiciones laborales en el trabajo docente. Digital Ciencia@UAQro,1(1),1-11. 
Tziner, A., y Sharoni, G. (2014). Organizational citizenship behavior, organizational justice, job stress, and work family conflict: Examination of their interrelationships with respondents from a non-Western culture. Journal of Work and Organizational Psychology, 30(1),35-42.

Tziner, A., Rabenu, E., Radomski, R., y Belkin, A. (2015). Work stress and turnover intentions among hospital physicians: The mediating role of burnout and work satisfaction. Journal of Work and Organizational Psychology, 31(1),207-2013.

Unda, S., Uribe, F., Jurado, S., García, M., Tovalín, H., y Juárez, A. (2016). Elaboración de una escala para valorar los factores de riesgo psicosocial en el trabajo de profesores universitarios. Journal of Work and Organizational Psychology,32(2), 67-74.

Valadez, A., Bravo, M., y Vaquero, J. (2014). Percepción de las fuentes de estrés laboral y sus efectos en el docente universitario. Revista Latinoamericana de Medicina Conductual.4(1), 21-31

\section{Notas}

[1] La organización mantiene el anonimato 